Proyecciones Journal of Mathematics

Vol. 38, No 2, pp. 295-304, June 2019.

Universidad Católica del Norte

Antofagasta - Chile

\title{
Total domination and vertex-edge domination in trees
}

\author{
Y. B. Venkatakrishnan \\ Sastra Deemed University, India \\ H. Naresh Kumar \\ Sastra Deemed University, India \\ and \\ C. Natarajan \\ Sastra Deemed University, India \\ Received : July 2017. Accepted : January 2019
}

\begin{abstract}
$A$ vertex $v$ of a graph $G=(V, E)$ is said to ve-dominate every edge incident to $v$, as well as every edge adjacent to these incident edges. A set $S \subseteq V$ is a vertex-edge dominating set if every edge of $E$ is ve-dominated by at least one vertex of $S$. The minimum cardinality of a vertex-edge dominating set of $G$ is the vertex-edge domination number $\gamma_{v e}(G)$. In this paper we prove $\left(\gamma_{t}(T)-\ell+1\right) / 2 \leq \gamma_{\mathrm{ve}}(\mathrm{T}) \leq$ $\left(\gamma_{\mathrm{t}}(\mathrm{T})+\ell-1\right) / 2$ and characterize trees attaining each of these bounds.
\end{abstract}

Keywords: Vertex-edge dominating set, total dominating set, trees.

AMS Subject Classification: $05 C 69$. 


\section{Introduction}

Let $G=(V, E)$ be a graph. By the neighborhood of a vertex $v$ of $G$ we mean the set $N_{G}(v)=\{u \in V(G): u v \in E(G)\}$. The degree of a vertex $v$, denoted by $d_{G}(v)$, is the cardinality of its neighborhood. By a leaf we mean a vertex of degree one, while a support vertex is a vertex adjacent to a leaf. The path on $\mathrm{n}$ vertices we denote by $P_{n}$. Let $T$ be a tree, and let $v$ be a vertex of $T$. We say that $v$ is adjacent to a path $P_{n}$ if there is a neighbor of $v$, say $x$, such that the subtree resulting from $T$ by removing the edge $v x$ and which contains the vertex $x$ as a leaf, is a path $P_{n}$.

A subset $D \subseteq V(G)$ is a dominating set, abbreviated DS, of $G$ if every vertex of $V(G) \backslash D$ has a neighbor in $D$. The domination number of a graph $G$, denoted by $\gamma(G)$, is the minimum cardinality of a dominating set of $G$. A subset $D \subseteq V(G)$ is a total dominating set, abbreviated TDS, of $G$ if every vertex of $V(G)$ has a neighbor in $D$. The total domination number of a graph $G$, denoted by $\gamma_{t}(G)$, is the minimum cardinality of a total dominating set of $G$. A total dominating set of $G$ of minimum cardinality is called a $\gamma_{t}(G)$-set. For more details on total domination, see [2].

An edge $e \in E(G)$ is vertex-edge dominated (ve-dominated) by a vertex $v \in V(G)$ if $e$ is incident to $v$, or $e$ is adjacent to an edge incident to $v$. A subset $D \subseteq V(G)$ is a vertex-edge dominating set, abbreviated VEDS, of $G$ if every edge of $G$ is vertex-edge dominated by a vertex of $D$. The vertex-edge domination number of $G$, denoted by $\gamma_{v e}(G)$, is the minimum cardinality of a vertex-edge dominating set of $G$. A vertex-edge dominating set of $G$ of minimum cardinality is called a $\gamma_{v e}(G)$-set. Vertex-edge domination in graphs was introduced in [5], and further studied in $[1,3,4]$.

In [4], trees with equal domination number and vertex-edge domination number are characterized. Here, we prove $\left(\gamma_{t}(T)-\ell+1\right) / 2 \leq \gamma_{\mathrm{ve}}(\mathrm{T}) \leq$ $\left(\gamma_{\mathrm{t}}(\mathrm{T})+\ell-1\right) / 2$ and characterize trees attaining each of these bounds.

\section{Main Results}

The one vertex graph does not have total dominating set and vertex-edge dominating set, in this paper, by a tree we mean only a connected graph with no cycle, and which has at least two vertices.

We begin with the following observations:

Observation 1. Every support vertex of a graph $G$ is in every TDS of graph $G$. 
Observation 2. Let $G$ be a graph and $u \in V(G)$. Let the vertex $u$ be adjacent to two paths $v w$ and $x y$. Let $v$ and $x$ be adjacent to $u$. Let $H=G-\{x, y\}$. Then $\gamma_{t}(G)=\gamma_{t}(H)+1$ and $\gamma_{v e}(G)=\gamma_{v e}(H)$.

Proof. Let $D$ be a $\gamma_{t}(G)$-set. By observation 1 , the vertices $v, x \in D$. To dominate the vertices $v$ and $x$, the vertex $u \in D$. It is easy to see that $D \backslash\{x\}$ is a TDS of the graph $H$. Thus $\gamma_{t}(H) \leq \gamma_{t}(G)-1$. Let $D^{\prime}$ be a $\gamma_{t}(H)$-set. By observation 1 , the vertex $v \in D^{\prime}$. To dominate $v$, the vertex $u \in D^{\prime}$. It is clear that $D^{\prime} \cup\{x\}$ is a TDS of the graph $G$. Thus $\gamma_{t}(G) \leq \gamma_{t}(H)+1$. We get $\gamma_{t}(G)=\gamma_{t}(H)+1$.

Let $D$ be a $\gamma_{v e}(T)$-set. To dominate the edges $x y$ and $v w$, the vertex $u \in D$. Obviously $D$ is a VEDS of the graph $H$. Thus $\gamma_{v e}(H) \leq \gamma_{v e}(G)$. Let $D^{\prime}$ be a $\gamma_{v e}(H)$-set. To dominate the edge $v w$, the vertex $u \in D^{\prime}$. Clearly the vertex $u$ dominates the edges $u x$ and $x y$ in the graph $G$. The set $D^{\prime}$ is a VEDS of the graph $G$. Thus $\gamma_{v e}(G) \leq \gamma_{v e}(H)$. We get $\gamma_{v e}(G)=\gamma_{v e}(H)$.

Observation 3. Let $H$ be a graph with a leaf $u$ adjacent to a weak support vertex $v$. Let $G$ be a graph obtained from $H$ by joining a path $P_{4}: x y z w$ to the leaf $u$. Let $u$ be adjacent to $x$. Then $\gamma_{t}(G)=\gamma_{t}(H)+2$ and $\gamma_{v e}(G)=\gamma_{v e}(H)+1$.

Proof. Let $D^{\prime}$ be a $\gamma_{t}(H)$-set. It is obvious that $D^{\prime} \cup\{z, y\}$ is a TDS of the graph $G$. Thus $\gamma_{t}(G) \leq \gamma_{t}(H)+2$. Let $D$ be a $\gamma_{t}(G)$-set. By observation $1, z \in D$. To dominate $z$, the vertex $y \in D$. It is clear that $D \backslash\{y, z\}$ is a TDS of the graph $H$. Thus $\gamma_{t}(H) \leq \gamma_{t}(G)-2$. We get $\gamma_{t}(G)=\gamma_{t}(H)+2$.

Let $D^{\prime}$ be a $\gamma_{v e}(H)$-set. It is clear that $D^{\prime} \cup\{y\}$ is a VEDS of the graph $G$. Thus $\gamma_{v e}(G) \leq \gamma_{v e}(H)+1$. Let $D$ be a $\gamma_{v e}(G)$-set. To dominate the edge $z w$, the vertex $y \in D$. It is obvious that $D \backslash\{y\}$ is a VEDS of the graph $H$. Thus $\gamma_{v e}(H) \leq \gamma_{v e}(G)-1$. We get $\gamma_{v e}(H)=\gamma_{v e}(G)-1$.

First we show that if $T$ is a nontrivial tree of order $n$ with $\ell$ leaves, then $\gamma_{v e}(T)$ is bounded below by $\left(\gamma_{t}(T)-\ell+1\right) / 2$. For the purpose of characterizing the trees attaining this bound we introduce a family $\mathcal{T}$ of trees $T=T_{k}$ that can be obtained as follows. Let $T_{1}=P_{5}$. If $k$ is a positive integer, then $T_{k+1}$ can be obtained recursively from $T_{k}$ by one of the following operations.

- Operation $\mathcal{O}_{1}$ : Attach a path $P_{2}$ by joining one of its vertices to a vertex of $T_{k}$ adjacent to a path $P_{2}$. 
- Operation $\mathcal{O}_{2}$ : Attach a path $P_{4}$ by joining one of its leaves to a leaf of $T_{k}$ whose support vertex is weak.

Lemma 4. If $T \in \mathcal{T}$, then $\gamma_{v e}(T)=\left(\gamma_{t}(T)-\ell+1\right) / 2$.

Proof. We use the induction on the number $k$ of operations performed to construct the tree $T$. If $T_{1}=P_{5}$, then $\gamma_{v e}\left(T_{1}\right)=1$ and $\gamma_{t}\left(T_{1}\right)=3$. It can be verified that $\gamma_{v e}\left(T_{1}\right)=\left(\gamma_{t}\left(T_{1}\right)-\ell+1\right) / 2$ is satisfied. Let $k \geq 2$ be an integer. Assume that the result is true for every tree $T^{\prime}=T_{k}$ of the family $\mathcal{T}$ constructed by $k-1$ operations. Let $\ell^{\prime}$ be the number of leaves of the tree $T^{\prime}$. Let $T=T_{k+1}$ be a tree of the family $\mathcal{T}$ constructed by $k$ operations.

First assume that $T$ is obtained from $T^{\prime}$ by operation $\mathcal{O}_{1}$. Let $x$ be a vertex to which a path $P_{2}=y z$ is attached. Let $x$ be adjacent to $y$. Let $u v$ be a path different from $y z$ attached at $x$. Let $u$ be adjacent to $x$. By observation $2, \gamma_{t}(T)=\gamma_{t}\left(T^{\prime}\right)+1$ and $\gamma_{v e}(T)=\gamma_{v e}\left(T^{\prime}\right)$. It is easy to see $\ell^{\prime}=\ell-1$. We get $\left(\gamma_{t}(T)-\ell+1\right) / 2=\left(\gamma_{\mathrm{t}}\left(\mathrm{T}^{\prime}\right)+1-\left(\ell^{\prime}+1\right)+1\right) / 2=$ $\gamma_{\mathrm{ve}}\left(\mathrm{T}^{\prime}\right)=\gamma_{\mathrm{ve}}(\mathrm{T})$.

Now assume that $T$ is obtained from $T^{\prime}$ by operation $\mathcal{O}_{2}$. Let $x$ be a leaf to which a path $P_{4}$ : uvwz is attached. Let $x$ be adjacent to $u$. By observation 3, $\gamma_{t}(T)=\gamma_{t}\left(T^{\prime}\right)+2$ and $\gamma_{v e}\left(T^{\prime}\right)=\gamma_{v e}(T)-1$. It is easy to see $\ell^{\prime}=\ell$. We get $\left(\gamma_{t}(T)-\ell+1\right) / 2=\left(\gamma_{\mathrm{t}}\left(\mathrm{T}^{\prime}\right)+2-\ell^{\prime}+1\right) / 2=$ $\left(\gamma_{\mathrm{t}}\left(\mathrm{T}^{\prime}\right)-\ell^{\prime}+1\right) / 2+1=\gamma_{\mathrm{ve}}\left(\mathrm{T}^{\prime}\right)+1=\gamma_{\mathrm{ve}}(\mathrm{T})$.

Theorem 5. If $T$ is a tree with $\ell$ leaves then $\gamma_{v e}(T) \geq\left(\gamma_{t}(T)-\ell+1\right) / 2$ with equality if and only if $T \in \mathcal{T}$.

Proof. If $\operatorname{diam}(T)=1$, then $T=P_{2}$. We have $\left(\gamma_{t}(T)-\ell+1\right) / 2=$ $(2-2+1) / 2<1=\gamma_{\mathrm{ve}}(\mathrm{T})$. If $\operatorname{diam}(T)=2$, then $T$ is a star $K_{1, n-1}$. We have $\left(\gamma_{t}(T)-\ell+1\right) / 2=(2-(\mathrm{n}-1)+1) / 2=(4-\mathrm{n}) / 2<1=\gamma_{\mathrm{ve}}(\mathrm{T})$ as $n \geq 3$. Now assume that $\operatorname{diam}(T) \geq 3$. Thus the order $n$ of the tree is at least four. We prove the result by induction on $n$. Assume that the theorem is true for every tree $T^{\prime}$ of order $n^{\prime}<n$ with $\ell^{\prime}$ leaves.

First assume that some support vertex of $T$, say $x$, is strong. Let $y$ and $z$ be two leaves adjacent to $x$. Let $T^{\prime}=T-y$. Let $D^{\prime}$ be a $\gamma_{t}\left(T^{\prime}\right)$-set. By observation $1, x \in D^{\prime}$. To dominate $x$, a vertex in $N_{G}(x)$ is in $D^{\prime}$. Clearly the vertex $x$ dominates $y$ in the tree $T$. The set $D^{\prime}$ is a TDS of the tree $T$. Thus $\gamma_{t}(T) \leq \gamma_{t}\left(T^{\prime}\right)$. It is clear that $\ell^{\prime}=\ell-1$. Let $D$ be a $\gamma_{v e}(T)$-set. It is obvious that $D$ is a VEDS of the tree $T^{\prime}$. Thus $\gamma_{v e}\left(T^{\prime}\right) \leq \gamma_{v e}(T)$. We now 
get $\left(\gamma_{t}(T)-\ell+1\right) / 2 \leq\left(\gamma_{\mathrm{t}}\left(\mathrm{T}^{\prime}\right)-\ell^{\prime}-1+1\right) / 2=\gamma_{\mathrm{ve}}\left(\mathrm{T}^{\prime}\right)-1 / 2<\gamma_{\mathrm{ve}}(\mathrm{T})$. Henceforth, every support vertex of $T$ is weak.

We now root $T$ at a vertex $r$ of maximum eccentricity $\operatorname{diam}(T)$. Let $t$ be a leaf at maximum distance from $r, v$ be the parent of $t$, and let $u$ be the parent of $v$ in the rooted tree. If $\operatorname{diam}(T) \geq 4$, then let $w$ be the parent of $u$. If $\operatorname{diam}(T) \geq 5$, then let $d$ be the parent of $w$. If $\operatorname{diam}(T) \geq 6$, then let $e$ be the parent of $d$. By $T_{x}$ we denote the subtree induced by a vertex $x$ and its descendants in the rooted tree.

From the last but one paragraph, we get $d_{T}(v)=2$. Assume that among the children of $u$ there is a support vertex, say $x$, other than $v$. Let $y$ be the leaf adjacent to $x$. Let $T^{\prime}=T-T_{x}$. We have $\ell^{\prime}=\ell-1$. Let $D^{\prime}$ be a $\gamma_{t}\left(T^{\prime}\right)$-set. By observation $1, v \in D^{\prime}$. To dominate $v$, the vertex $u \in D^{\prime}$. Clearly $D^{\prime} \cup\{x\}$ is a TDS of the tree $T$. Thus $\gamma_{t}(T) \leq \gamma_{t}\left(T^{\prime}\right)+1$. Let $D$ be a $\gamma_{v e}(T)$-set. To dominate the edge $v t$ and $x y$, the vertex $u \in D$. Clearly $D$ is a VEDS of the tree $T^{\prime}$. Thus $\gamma_{v e}\left(T^{\prime}\right) \leq \gamma_{v e}(T)$. We now get $\left(\gamma_{t}(T)-\ell+1\right) / 2 \leq\left(\gamma_{\mathrm{t}}\left(\mathrm{T}^{\prime}\right)+1-\ell^{\prime}-1+1\right) / 2=\gamma_{\mathrm{ve}}\left(\mathrm{T}^{\prime}\right) \leq \gamma_{\mathrm{ve}}(\mathrm{T})$. This implies that $\left(\gamma_{t}\left(T^{\prime}\right)-\ell^{\prime}+1\right) / 2=\gamma_{\mathrm{ve}}\left(\mathrm{T}^{\prime}\right)$. By the induction hypothesis we have $T^{\prime} \in \mathcal{T}$. The tree $T$ can be obtained from $T^{\prime}$ by operation $\mathcal{O}_{1}$. Thus $T \in \mathcal{T}$.

Assume that among the children of $u$, other than $v$, there is a leaf $x$. Let $T^{\prime}=T-x$. We have $\ell=\ell^{\prime}+1$. Let $D^{\prime}$ be a $\gamma_{t}\left(T^{\prime}\right)$-set. By observation 1 , $v \in D^{\prime}$. To dominate $v$, the vertex $u \in D^{\prime}$. Clearly $D^{\prime}$ is a TDS of the tree $T$. Thus $\gamma_{t}(T) \leq \gamma_{t}\left(T^{\prime}\right)$. Let $D$ be a $\gamma_{v e}(T)$-set. To dominate the edge $v t$, the vertex $u \in D$. It is easy to see that $D$ is a VEDS of the tree $T^{\prime}$. Thus $\gamma_{v e}\left(T^{\prime}\right) \leq \gamma_{v e}(T)$. We now get $\left(\gamma_{t}(T)-\ell+1\right) / 2 \leq\left(\gamma_{\mathrm{t}}\left(\mathrm{T}^{\prime}\right)-\ell^{\prime}-1+1\right) / 2=$ $\gamma_{\mathrm{ve}}\left(\mathrm{T}^{\prime}\right)-1 / 2<\gamma_{\mathrm{ve}}(\mathrm{T})$.

We assume that $d_{T}(u)=2$. Now assume that among the children of $w$, other than $u$, there is a vertex $x$ such that the distance of $w$ to the most distant vertex of $T_{x}$ is three. It suffices to consider that $w$ is adjacent to a path $P_{3}: x y z$. Let $T^{\prime}=T-T_{u}$. We have $\ell=\ell^{\prime}+1$. Let $D^{\prime}$ be a $\gamma_{t}\left(T^{\prime}\right)$-set. It is easy to see that $D^{\prime} \cup\{u, v\}$ is a TDS of the tree $T$. Thus $\gamma_{t}(T) \leq \gamma_{t}\left(T^{\prime}\right)+2$. Let $D$ be a $\gamma_{v e}(T)$-set. To dominate the edges $v t$ and $y z$, the vertices $u, x \in D$. It is easy to observe that $D \backslash\{u\}$ is a VEDS of the tree $T^{\prime}$. Thus $\gamma_{v e}\left(T^{\prime}\right) \leq \gamma_{v e}(T)-1$. We now get $\left(\gamma_{t}(T)-\ell+1\right) / 2 \leq\left(\gamma_{\mathrm{t}}\left(\mathrm{T}^{\prime}\right)+2-\ell^{\prime}-1+1\right) / 2=\gamma_{\mathrm{ve}}\left(\mathrm{T}^{\prime}\right)+1 / 2<\gamma_{\mathrm{ve}}(\mathrm{T})$.

Assume that among the children of $w$, other than $u$, there is a vertex $x$ such that the distance of $w$ to the most distant vertex of $T_{x}$ is two. It suffices to consider that $w$ is adjacent to a path $P_{2}: x y$. Let $T^{\prime}=T-T_{u}$. We have $\ell=\ell^{\prime}+1$. Let $D^{\prime}$ be a $\gamma_{t}\left(T^{\prime}\right)$-set. It is easy to observe that $D^{\prime} \cup\{u, v\}$ 
is a TDS of the tree $T$. Thus $\gamma_{t}(T) \leq \gamma_{t}\left(T^{\prime}\right)+2$. Let $D$ be a $\gamma_{v e}(T)$-set. To dominate the edges $v t$ and $y x$, the vertices $u, w \in D$. It is clear that $D \backslash\{u\}$ is a VEDS of the tree $T^{\prime}$. Thus $\gamma_{v e}\left(T^{\prime}\right) \leq \gamma_{v e}(T)-1$. We now get $\left(\gamma_{t}(T)-\ell+1\right) / 2 \leq\left(\gamma_{\mathrm{t}}\left(\mathrm{T}^{\prime}\right)+2-\ell^{\prime}-1+1\right) / 2=\gamma_{\mathrm{ve}}\left(\mathrm{T}^{\prime}\right)+1 / 2<\gamma_{\mathrm{ve}}(\mathrm{T})$.

Assume that among the children of $w$, other than $u$, there is a leaf $x$. Let $T^{\prime}=T-T_{w}$. We have $\ell=\ell^{\prime}+2$. Let $D^{\prime}$ be a $\gamma_{t}\left(T^{\prime}\right)$-set. It is easy to see that $D^{\prime} \cup\{u, v, w\}$ is a TDS of the tree $T$. Thus $\gamma_{t}(T) \leq \gamma_{t}\left(T^{\prime}\right)+3$. Let $D$ be a $\gamma_{v e}(T)$-set. To dominate the edges $v t, u v, u w, w x$ and $w d$, the vertex $u \in D$. It is clear that $D \backslash\{u\}$ is a VEDS of the tree $T^{\prime}$. Thus $\gamma_{v e}\left(T^{\prime}\right) \leq \gamma_{v e}(T)-1$. We now get $\left(\gamma_{t}(T)-\ell+1\right) / 2 \leq\left(\gamma_{\mathrm{t}}\left(\mathrm{T}^{\prime}\right)+3-\ell^{\prime}-2+1\right) / 2=\gamma_{\mathrm{ve}}\left(\mathrm{T}^{\prime}\right)+1 / 2<$ $\gamma_{\mathrm{ve}}(\mathrm{T})$.

Assume $d_{T}(d) \geq 3$. Let $T^{\prime}=T-T_{w}$. We have $\ell=\ell^{\prime}+1$. Let $D^{\prime}$ be a $\gamma_{t}\left(T^{\prime}\right)$-set. It is clear that $D^{\prime} \cup\{u, v\}$ is a TDS of the tree $T$. Thus $\gamma_{t}(T) \leq \gamma_{t}\left(T^{\prime}\right)+2$. Let $D$ be a $\gamma_{v e}(T)$-set. To dominate the edges $v t, u v, u w$ and $w d$, the vertex $u \in D$. It is clear that $D \backslash\{u\}$ is a VEDS of the tree $T^{\prime}$. Thus $\gamma_{v e}\left(T^{\prime}\right) \leq \gamma_{v e}(T)-1$. We now get $\left(\gamma_{t}(T)-\ell+1\right) / 2 \leq$ $\left(\gamma_{\mathrm{t}}\left(\mathrm{T}^{\prime}\right)+2-\ell^{\prime}-1+1\right) / 2=\gamma_{\mathrm{ve}}\left(\mathrm{T}^{\prime}\right)+1 / 2<\gamma_{\mathrm{ve}}(\mathrm{T})$.

Suppose $d=r$, we have $T=P_{5}=$ rwuvt. Then $\left(\gamma_{t}(T)-\ell+1\right) / 2=$ $(3-2+1) / 2=1=\gamma_{\mathrm{ve}}(\mathrm{T})$. Thus $T \in \mathcal{T}$. Now assume that $d_{T}(d)=2$. Let $T^{\prime}=T-T_{w}$. We have $\ell=\ell^{\prime}$. Placing the arguments as in the previous case, we get $\left(\gamma_{t}(T)-\ell+1\right) / 2 \leq\left(\gamma_{\mathrm{t}}\left(\mathrm{T}^{\prime}\right)+2-\ell^{\prime}+1\right) / 2 \leq \gamma_{\mathrm{ve}}\left(\mathrm{T}^{\prime}\right)+1 \leq \gamma_{\mathrm{ve}}(\mathrm{T})$. This implies that $\left(\gamma_{t}\left(T^{\prime}\right)-\ell^{\prime}+1\right) / 2=\gamma_{\mathrm{ve}}\left(\mathrm{T}^{\prime}\right)$. By the inductive hypothesis $T^{\prime} \in \mathcal{T}$. The tree $T$ can be obtained from $T^{\prime}$ by operation $\mathcal{O}_{2}$. Thus $T \in \mathcal{T}$.

We now show that if $T$ is a nontrivial tree of order $n$ with $\ell$ leaves, then $\gamma_{v e}(T)$ is bounded above by $\left(\gamma_{t}(T)+\ell-2\right) / 2$. For the purpose of characterizing the trees attaining this bound we introduce a family $\mathcal{F}$ of trees $T=T_{k}$ that can be obtained as follows. Let $T_{1} \in\left\{P_{2}, P_{3}, P_{4}\right\}$. If $k$ is a positive integer, then $T_{k+1}$ can be obtained recursively from $T_{k}$ by operation $\mathrm{O}_{2}$.

It is easy to see that $\mathcal{F}$ consists of paths $P_{n}$ where $n \neq 4 k+1$ for positive integer $k$.

Lemma 6. If $T \in \mathcal{T}$, then $\gamma_{v e}(T)=\left(\gamma_{t}(T)+\ell-2\right) / 2$.

Proof. We use the induction on the number $k$ of operations performed to construct the tree $T$. If $T_{1} \in\left\{P_{2}, P_{3}, P_{4}\right\}$, then $\gamma_{v e}\left(T_{1}\right)=1$ and $\gamma_{t}\left(T_{1}\right)=2$. It can be verified that $\gamma_{v e}\left(T_{1}\right)=\left(\gamma_{t}\left(T_{1}\right)+\ell-2\right) / 2$ is satisfied. Let $k \geq 2$ be an integer. Assume that the result is true for every tree $T^{\prime}=T_{k}$ of the 
family $\mathcal{F}$ constructed by $k-1$ operations. Let $\ell^{\prime}$ be the number of leaves of the tree $T^{\prime}$. Let $T=T_{k+1}$ be a tree of the family $\mathcal{F}$ constructed by $k$ operations.

Assume that $T$ is obtained from $T^{\prime}$ by operation $\mathcal{O}_{2}$. Let $x$ be a leaf to which a path $P_{4}: u v w z$ is attached. Let $x$ be adjacent to $u$. By observation $3, \gamma_{t}(T)=\gamma_{t}\left(T^{\prime}\right)+2$ and $\gamma_{v e}\left(T^{\prime}\right)=\gamma_{v e}(T)-1$. It is easy to see $\ell^{\prime}=\ell$. We get $\left(\gamma_{t}(T)+\ell-2\right) / 2=\left(\gamma_{\mathrm{t}}\left(\mathrm{T}^{\prime}\right)+2+\ell^{\prime}-2\right) / 2=\left(\gamma_{\mathrm{t}}\left(\mathrm{T}^{\prime}\right)+\ell^{\prime}-2\right) / 2+1=$ $\gamma_{\mathrm{ve}}\left(\mathrm{T}^{\prime}\right)+1=\gamma_{\mathrm{ve}}(\mathrm{T})$.

Theorem 7. If $T$ is a tree with $\ell$ leaves then $\gamma_{v e}(T) \leq\left(\gamma_{t}(T)+\ell-2\right) / 2$ with equality if and only if $T \in \mathcal{F}$.

Proof. If $\operatorname{diam}(T)=1$, then $T=P_{2}$. We have $\left(\gamma_{t}(T)+\ell-2\right) / 2=$ $(2+2-2) / 2=1=\gamma_{\mathrm{ve}}(\mathrm{T})$. Thus $T \in \mathcal{F}$. If $\operatorname{diam}(T)=2$. If $T$ is a path $P_{3}$, we have $\left(\gamma_{t}(T)+\ell-2\right) / 2=(2+2-2) / 2=1=\gamma_{\mathrm{ve}}(\mathrm{T})$. Thus $T \in \mathcal{F}$. $T$ is a star $K_{1, n-1}$ other than $P_{3}$. We have $\left(\gamma_{t}(T)+\ell-2\right) / 2=$ $(2+(\mathrm{n}-1)-2) / 2=(\mathrm{n}-1) / 2>1=\gamma_{\mathrm{ve}}(\mathrm{T})$ as $n>3$. Now assume that $\operatorname{diam}(T) \geq 3$. Thus the order $n$ of the tree is at least four. We prove the result by induction on $n$. Assume that the theorem is true for every tree $T^{\prime}$ of order $n^{\prime}<n$ with $\ell^{\prime}$ leaves.

First assume that some support vertex of $T$, say $x$, is strong. Let $y$ and $z$ be two leaves adjacent to $x$. Let $T^{\prime}=T-y$. We have $\ell^{\prime}=\ell-1$. Let $D^{\prime}$ be a $\gamma_{v e}\left(T^{\prime}\right)$-set. The vertex which dominates the edge $x z$ in the tree $T^{\prime}$ dominates the edge $x y$ in the tree $T$. It is obvious that $D^{\prime}$ is a VEDS of the tree $T$. Thus $\gamma_{v e}(T) \leq \gamma_{v e}\left(T^{\prime}\right)$. Let $D$ be a $\gamma_{t}(T)$-set. By observation $1, x \in D$. To dominate $x$, a vertex in $N_{G}(x)$ is in $D$. If $y \in D$ then $(D \backslash\{y\}) \cup\{z\}$ is a TDS of the tree $T^{\prime}$. Thus $\gamma_{t}\left(T^{\prime}\right) \leq \gamma_{t}(T)$. We now get $\gamma_{v e}(T) \leq \gamma_{v e}\left(T^{\prime}\right) \leq\left(\gamma_{t}\left(T^{\prime}\right)+\ell^{\prime}-2\right) / 2 \leq\left(\gamma_{\mathrm{t}}(\mathrm{T})+\ell-1-2\right) / 2<$ $\left(\gamma_{\mathrm{t}}(\mathrm{T})+\ell-2\right) / 2$. Henceforth, every support vertex of $T$ is weak.

We now root $T$ at a vertex $r$ of maximum eccentricity $\operatorname{diam}(T)$. Let $t$ be a leaf at maximum distance from $r, v$ be the parent of $t$, and let $u$ be the parent of $v$ in the rooted tree. If $\operatorname{diam}(T) \geq 4$, then let $w$ be the parent of $u$. If $\operatorname{diam}(T) \geq 5$, then let $d$ be the parent of $w$. If $\operatorname{diam}(T) \geq 6$, then let $e$ be the parent of $d$. By $T_{x}$ we denote the subtree induced by a vertex $x$ and its descendants in the rooted tree.

Assume that among the children of $u$, other than $v$, there is a leaf $x$. Let $T^{\prime}=T-x$. We have $\ell=\ell^{\prime}+1$. Let $D^{\prime}$ be a $\gamma_{v e}\left(T^{\prime}\right)$-set. To dominate the edge $v t$, the vertex $u \in D^{\prime}$. Clearly $D^{\prime}$ is a VEDS of the tree $T$ as $u$ dominates the edge $u x$. Thus $\gamma_{v e}(T) \leq \gamma_{v e}\left(T^{\prime}\right)$. Let $D$ be a $\gamma_{t}(T)$-set. By 
observation 1 , the vertices $u, v \in D$. It is obvious that $D$ is a TDS of the tree $T^{\prime}$. Thus $\gamma_{t}\left(T^{\prime}\right) \leq \gamma_{t}(T)$. We get $\gamma_{v e}(T) \leq \gamma_{v e}\left(T^{\prime}\right) \leq\left(\gamma_{t}\left(T^{\prime}\right)+\ell^{\prime}-2\right) / 2 \leq$ $\left(\gamma_{\mathrm{t}}(\mathrm{T})+\ell-1-2\right) / 2<\left(\gamma_{\mathrm{t}}(\mathrm{T})+\ell-2\right) / 2$.

Assume that among the children of $u$ there is a support vertex, say $x$, other than $v$. Let $y$ be the leaf adjacent to $x$. Let $T^{\prime}=T-T_{x}$. We have $\ell^{\prime}=\ell-1$. Let $D^{\prime}$ be a $\gamma_{v e}\left(T^{\prime}\right)$-set. To dominate the edge $v t$, the vertex $u \in D^{\prime}$. The vertex $u$ dominates the edges $u x$ and $x y$ in the tree $T$. Clearly $D^{\prime}$ is a VEDS of the tree $T$. Thus $\gamma_{v e}(T) \leq \gamma_{v e}\left(T^{\prime}\right)$. Let $D$ be a $\gamma_{t}(T)$-set. By observation 1 , the vertices $v, x \in D$. To dominate $v$ and $x$, the vertex $u \in D$. It is obvious that $D \backslash\{x\}$ is a TDS of the tree $T^{\prime}$. Thus $\gamma_{t}\left(T^{\prime}\right) \leq \gamma_{t}(T)-1$. We get $\gamma_{v e}(T) \leq \gamma_{v e}\left(T^{\prime}\right) \leq\left(\gamma_{t}\left(T^{\prime}\right)+\ell^{\prime}-2\right) / 2 \leq$ $\left(\gamma_{\mathrm{t}}(\mathrm{T})-1+\ell-1-2\right) / 2<\left(\gamma_{\mathrm{t}}(\mathrm{T})+\ell-2\right) / 2$.

We assume that $d_{T}(u)=2$. Now assume that among the children of $w$, other than $u$, there is a vertex $x$ such that the distance of $w$ to the most distant vertex of $T_{x}$ is three. It suffices to consider that $w$ is adjacent to a path $P_{3}$ : xyz. Let $T^{\prime}=T-T_{u}$. We have $\ell=\ell^{\prime}+1$. Let $D^{\prime}$ be a $\gamma_{v e}\left(T^{\prime}\right)$-set. It is obvious that $D^{\prime} \cup\{u\}$ is a VEDS of the tree $T$. Thus $\gamma_{v e}(T) \leq \gamma_{v e}\left(T^{\prime}\right)+1$. Let $D$ be a $\gamma_{t}(T)$-set. By observation 1 , the vertices $y, v \in D$. To dominate the two vertices $y$ and $v$ the vertices $x, u \in D$. It is clear that $D \backslash\{u, v\}$ is a TDS of the tree $T^{\prime}$. Thus $\gamma_{t}\left(T^{\prime}\right) \leq \gamma_{t}(T)-2$. We get $\gamma_{v e}(T) \leq \gamma_{v e}\left(T^{\prime}\right)+1 \leq\left(\gamma_{t}\left(T^{\prime}\right)+\ell^{\prime}-2\right) / 2+1 \leq\left(\gamma_{\mathrm{t}}(\mathrm{T})-2+\ell-1-2\right) / 2+1<$ $\left(\gamma_{\mathrm{t}}(\mathrm{T})+\ell-2\right) / 2$.

Assume that among the children of $w$, other than $u$, there is a vertex $x$ such that the distance of $w$ to the most distant vertex of $T_{x}$ is two. It suffices to consider that $w$ is adjacent to a path $P_{2}: x y$. Let $T^{\prime}=T-T_{u}$. We have $\ell=\ell^{\prime}+1$. Let $D^{\prime}$ be a $\gamma_{v e}\left(T^{\prime}\right)$-set. It is obvious that $D^{\prime} \cup\{u\}$ is a VEDS of the tree $T$. Thus $\gamma_{v e}(T) \leq \gamma_{v e}\left(T^{\prime}\right)+1$. Let $D$ be a $\gamma_{t}(T)$-set. By observation 1 , the vertices $x, v \in D$. To dominate $x, v$ the vertices $w, u \in D$. It is clear that $D \backslash\{u, v\}$ is a TDS of the tree $T^{\prime}$. Thus $\gamma_{t}\left(T^{\prime}\right) \leq \gamma_{t}(T)-2$. We get $\gamma_{v e}(T) \leq \gamma_{v e}\left(T^{\prime}\right)+1 \leq\left(\gamma_{t}\left(T^{\prime}\right)+\ell^{\prime}-2\right) / 2+1 \leq\left(\gamma_{\mathrm{t}}(\mathrm{T})-2+\ell-1-2\right) / 2+1<$ $\left(\gamma_{\mathrm{t}}(\mathrm{T})+\ell-2\right) / 2$.

Assume that among the children of $w$, other than $u$, there is a leaf $x$. Let $T^{\prime}=T-T_{w}$. We have $\ell=\ell^{\prime}+2$. Let $D^{\prime}$ be a $\gamma_{v e}\left(T^{\prime}\right)$-set. It is obvious that $D^{\prime} \cup\{u\}$ is a VEDS of the tree $T$. Thus $\gamma_{v e}(T) \leq \gamma_{v e}\left(T^{\prime}\right)+1$. Let $D$ be a $\gamma_{t}(T)$-set. By observation 1 , the vertices $v, w \in D$. To dominate $v$ and $w$, the vertex $u \in D$. It is obvious that $(D \backslash\{w, u, v\}) \cup\{a\}$ where $a$ is a vertex in $N_{G}(d)$ other than $w$ is a TDS of the tree $T^{\prime}$. Thus $\gamma_{t}\left(T^{\prime}\right) \leq \gamma_{t}(T)-2$. We get $\gamma_{v e}(T) \leq \gamma_{v e}\left(T^{\prime}\right)+1 \leq\left(\gamma_{t}\left(T^{\prime}\right)+\ell^{\prime}-2\right) / 2+1 \leq\left(\gamma_{t}(\mathrm{~T})-2+\ell-2-2\right) / 2+1<$ $\left(\gamma_{\mathrm{t}}(\mathrm{T})+\ell-2\right) / 2$. 
Assume $d_{T}(d) \geq 3$. Let $T^{\prime}=T-T_{w}$. We have $\ell=\ell^{\prime}+1$. Let $D^{\prime}$ be a $\gamma_{v e}\left(T^{\prime}\right)$-set. It is obvious that $D^{\prime} \cup\{u\}$ is a VEDS of the tree $T$. Thus $\gamma_{v e}(T) \leq \gamma_{v e}\left(T^{\prime}\right)+1$. Let $D$ be a $\gamma_{t}(T)$-set. By observation $1, v \in D$. To dominate $v$, the vertex $u \in D$. It is clear that $D \backslash\{u, v\}$ is a TDS of the tree $T^{\prime}$. Thus $\gamma_{t}\left(T^{\prime}\right) \leq \gamma_{t}(T)-2$. We get $\gamma_{v e}(T) \leq \gamma_{v e}\left(T^{\prime}\right)+1 \leq$ $\left(\gamma_{t}\left(T^{\prime}\right)+\ell^{\prime}-2\right) / 2+1 \leq\left(\gamma_{\mathrm{t}}(\mathrm{T})-2+\ell-1-2\right) / 2+1<\left(\gamma_{\mathrm{t}}(\mathrm{T})+\ell-2\right) / 2$.

Now assume $d_{T}(d)=2$. Let $T^{\prime}=T-T_{w}$. We have $\ell=\ell^{\prime}$. Let $D^{\prime}$ be a $\gamma_{v e}\left(T^{\prime}\right)$-set. It is obvious that $D^{\prime} \cup\{u\}$ is a VEDS of the tree $T$. Thus $\gamma_{v e}(T) \leq \gamma_{v e}\left(T^{\prime}\right)+1$. Let $D$ be a $\gamma_{t}(T)$-set. By observation $1, v \in D$. To dominate $v$, the vertex $u \in D$. It is clear that $D \backslash\{u, v\}$ is a TDS of the tree $T^{\prime}$. Thus $\gamma_{t}\left(T^{\prime}\right) \leq \gamma_{t}(T)-2$. We get $\gamma_{v e}(T) \leq \gamma_{v e}\left(T^{\prime}\right)+1 \leq$ $\left(\gamma_{t}\left(T^{\prime}\right)+\ell^{\prime}-2\right) / 2+1 \leq\left(\gamma_{\mathrm{t}}(\mathrm{T})-2+\ell-2\right) / 2+1 \leq\left(\gamma_{\mathrm{t}}(\mathrm{T})+\ell-2\right) / 2$. This implies that $\gamma_{v e}\left(T^{\prime}\right)=\left(\gamma_{t}\left(T^{\prime}\right)+\ell^{\prime}-2\right) / 2$. By the inductive hypothesis $T^{\prime} \in \mathcal{T}$. The tree $T$ can be obtained from $T^{\prime}$ by operation $\mathcal{O}_{2}$. Thus $T \in \mathcal{T}$.

Acknowledgement: The authors thank the learned reviewers for their suggestions which improved the presentation of the paper. The first author thank National Board of Higher Mathematics, India for the support towards this work (NBHM/R.P.1/2015/Fresh/168) and to Department of Science and Technology, Government of India for the financial support to the Mathematics department SASTRA University under FIST Programme SR/FST/MSI-107/2015.

\section{References}

[1] R. Boutrig, M. Chellali, T. W. Haynes and S. T. Hedetniemi, Vertexedge domination in graphs. Aequat. Math., 90, pp. 355-366, (2016).

[2] M. A. Henning and A. Yeo, Total Domination in Graphs (Springer Monographs in Mathematics). 2013. ISBN: 978-1-4614-6524-9 (Print) 978-1-4614-6525-6 (Online).

[3] B. Krishnakumari, Y. B. Venkatakrishnan and M. Krzywkowski, Bounds on the vertex-edge domination number of a tree. C. R. Acad. Sci. Paris, Ser.I 352, pp. 363-366, (2014).

[4] J. R. Lewis, S. T. Hedetniemi, T. W. Haynes and G. H. Fricke, Vertexedge domination. Util. Math. 81, pp. 193-213, (2010). 
304 Y. B. Venkatakrishnan, H. Naresh Kumar and C. Natarajan

[5] J. W. Peters. Theoretical and algorithmic results on domination and connectivity. Ph.D. Thesis, Clemson University, (1986).

\section{Y. B. Venkatakrishnan}

Department of Mathematics, Sastra Deemed University

Tanjore, Tamilnadu,

India

e-mail: ybvenkatakrishnan2@gmail.com

\section{H. Naresh Kumar}

Department of Mathematics, Sastra Deemed University

Tanjore, Tamilnadu,

India

e-mail: nareshhari1403@gmail.com

and

\section{Natarajan}

Department of Mathematics,

Sastra Deemed University

Tanjore, Tamilnadu,

India

e-mail: natarajan@maths.sastra.edu 